EPJ Web of Conferences 37, 04002 (2012)

DOI: $10.1051 /$ epjconf/20123704002

C) Owned by the authors, published by EDP Sciences, 2012

\title{
Interesting features of scalar and pseudoscalar spectral function sum rules within a $U(3)$ chiral framework
}

\author{
$\underline{\text { Zhi-Hui Guo }}^{1,2}$, J. A. Oller ${ }^{2}$, and J. Ruiz de Elvira ${ }^{3}$
}

1 Department of Physics, Hebei Normal University, 050024 Shijiazhuang, P. R. China

2 Departamento de Física, Universidad de Murcia, E-30071 Murcia, Spain

3 Departamento de Física Teórica II, Universidad Complutense de Madrid, E-28040 Madrid, Spain

\begin{abstract}
We compute the scalar and pseudoscalar spectral functions by using their form factors calculated within the unitarized $U(3)$ chiral perturbation theory $(\chi \mathrm{PT})$. The extrapolation to larger values of $N_{C}$ and the spectral function sum rules are discussed as well. Some interesting features of the scalar spectral functions are revealed.
\end{abstract}

\section{Introduction}

Spectral function or the absorptive part of two-point current-current correlator plays important roles in hadron physics. It may provide a bridge to connect the asymptotic region of QCD made of quarks and gluons with the nonperturbative region of QCD made of various hadrons [1]. In the asymptotic energy regime, operator production expansion (OPE) supplies a reliable way to compute the currentcurrent correlators, where the perturbative dynamics is collected in the Wilson coefficients and the nonperturbative effects are represented by the non-vanishing operator condensates. Nevertheless to know the values of different operator condensates, one needs to connect the OPE results with the experimental observables, which usually involve hadronic degrees of freedom. Hence inevitably, we need to calculate the spectral functions in the nonperturbative energy region as well. The common way to construct the spectral functions is the resonances + continuum method [1], where the resonances are typically introduced by using the narrow width approximation. Though this approach to evaluate the spectral functions is quite simple and efficient, it may oversimplify the underlying dynamics in the nonperturbative QCD region. For examples, there exist very broad resonances, such as $\sigma$ and $\kappa$ [2], for which the narrow width approximation clearly does not work, and also the situation becomes complicated when the resonances and nearby thresholds meet together.

On the other hand, $\chi \mathrm{PT}$ has been proven to be a powerful and reliable approach to handle the physics in low energy QCD [3]. However, $\chi$ PT, whose success is based on momentum and light quark mass perturbative expansions, is not valid any more in the energy region where resonance states appear. A sophisticated approach, unitarized $\chi \mathrm{PT}$ that inherits the chiral symmetry and also extends the applicable energy region of $\chi \mathrm{PT}$ by resumming the unitarity cut, is then brought up and has been demonstrated useful in many processes [4-10]. Therefore, unitarized $\chi$ PT can provide us another reliable way to calculate the spectral functions. In our recent works, Refs. [7-10], we first fit the free parameters in our theory to meson-meson scattering data and then scalar and pseudoscalar spectral functions calculated within the framework of unitarized $U(3) \chi \mathrm{PT}$ with explicit exchanges of resonances are predicted. In this note, we briefly review our main results on the spectral functions obtained in Refs. [7-10]. unrestricted use, distribution, and reproduction in any medium, provided the original work is properly cited. 


\section{Theoretical formalism}

Our theoretical bases to calculate the scalar and pesudoscalar spectral functions are the $U(3) \chi \mathrm{PT}$ Lagrangian [11], resonance chiral theory [12] and the simplified N/D approach for unitarization [4, 5]. The scalar spectral function, or the imaginary part of the scalar-density-scalar-density correlator, is constructed by using the corresponding scalar form factors

$$
\operatorname{Im} \Pi_{S^{a}}(s)=\sum_{i} \rho_{i}(s)\left|F_{i}^{a}(s)\right|^{2} \theta\left(s-s_{i}^{\mathrm{th}}\right),
$$

where the index $i$ labels all the different pseudo-Goldstone boson pairs $P Q$ in $U(3) \chi \mathrm{PT}$ with the same quantum numbers as the considered spectral function, $\theta(x)$ is the Heaviside step function, the threshold of the $P Q$ meson pair in the $i_{\text {th }}$-channel reads $s_{i}^{\text {th }}=\left(m_{P}+m_{Q}\right)^{2}$ and $\rho_{i}(s)=\frac{\sqrt{\left[s-\left(m_{P}+m_{Q}\right)^{2}\right]\left[s-\left(m_{P}-m_{Q}\right)^{2}\right]}}{16 \pi s}$ is a phase-space factor. The scalar form factor of the $P Q$ pair in the $i_{t h}$ channel in Eq. (1) is defined as

$$
F_{P Q}^{a}(s)=\frac{1}{B}\left\langle 0\left|\bar{q} \lambda_{a} q\right| P Q\right\rangle,
$$

where $B$ is related to the quark condensates in the chiral and large $N_{C}$ limits [7], $\lambda_{a}$ with $a=1,2,3, \ldots, 8$ are the standard Gell-Mann matrices and $\lambda_{0}=\sqrt{\frac{2}{3}} I_{3 \times 3}$ with $I_{3 \times 3}$ the $3 \times 3$ unit matrix. We concentrate on the strangeness conserving cases, i.e. $a=0,8,3$. In the N/D approach [4,5], the unitarized scalar form factor is related to the perturbative results of the scalar form factors and meson-meson scattering amplitudes through

$$
F_{\mathrm{U}}^{I}(s)=\left[1+N^{I J}(s) g^{I J}(s)\right]^{-1} R^{I}(s),
$$

where $F_{\mathrm{U}}^{I}(s)$ stands for the unitarized scalar form factor with isospin $I$ and $R^{I}(s)=F^{I}(s)^{(2)+\text { Res+Loop }}+$ $T^{I J}(s)^{(2)} g^{I J}(s) F^{I}(s)^{(2)}$. Here $F^{I}(s)^{(2)+\text { Res+Loop }}$ denotes the perturbative calculation of the scalar form factors, $g^{I J}(s)$ collects the unitarity cuts, $N^{I J}(s)$ contains the crossed-channel cuts from meson-meson scattering amplitudes and $T^{I J}(s)^{(2)}$ corresponds to the leading order scattering amplitudes. We refer to Refs. [7,9] for details.

We only include the single-particle exchange contributions from either pseudo-Goldstone bosons or pseudoscalar resonances to calculate the pseudoscalar spectral functions

$$
\operatorname{Im} \Pi_{P^{a}}(s)=\sum_{j} \pi \delta\left(s-m_{P_{j}}^{2}\right)\left|H_{j}^{a}(s)\right|^{2} .
$$

In the previous equation $j$ stands for the pseudo-Goldstone bosons or pseudoscalar resonances with the same quantum numbers as the considered spectral functions and the pseudoscalar form factors are defined as $H_{P}^{a}(s)=\frac{1}{B}\left\langle 0\left|i \bar{q} \gamma_{5} \lambda_{a} q\right| P\right\rangle$. The explicit formulas for the scattering amplitudes and form factors can be found in Refs. [7,9].

The spectral function sum rules we shall study apply in the chiral limit and are given by

$$
\int_{0}^{s_{0}}\left[\operatorname{I} m \Pi_{R}(s)-\operatorname{I} m \Pi_{R^{\prime}}(s)\right] d s+\int_{s_{0}}^{\infty}\left[\operatorname{Im} \Pi_{R}(s)-\operatorname{I} m \Pi_{R^{\prime}}(s)\right] d s=0,
$$

with $R, R^{\prime}=S$ or $P$. Notice that according to the OPE results from Ref. [13] the second integral in the above equation vanishes in the chiral limit for all the scalar and pseudoscalar spectral functions considered in our work. Then we only need to focus on the nonperturbative dynamics of different spectral functions represented by the first integral.

\section{Results and discussions}

We would like to mention that all free parameters in the scalar and pseudoscalar spectral functions in our theory already appear in meson-meson scattering. Therefore, we first fit the free parameters to 


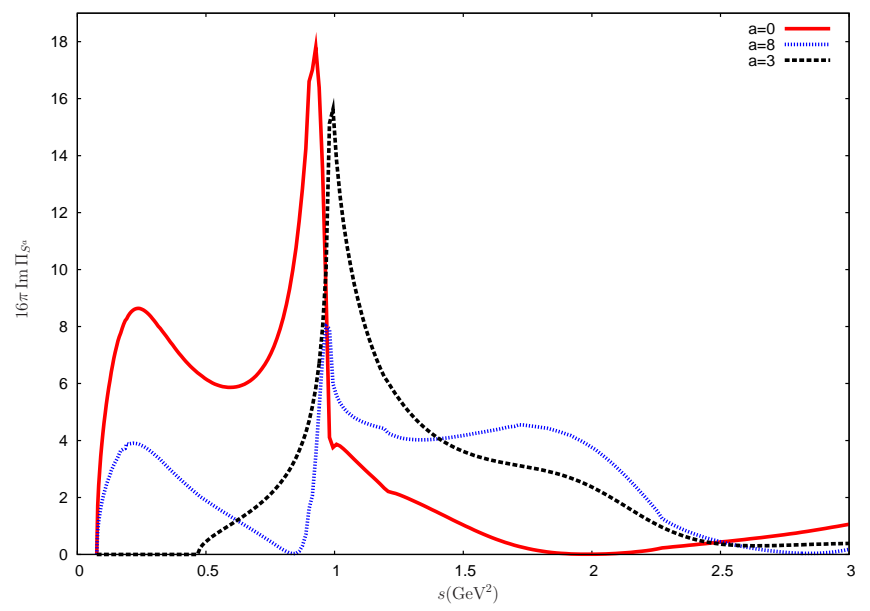

Fig. 1. Scalar spectral functions with physical masses of light mesons at $N_{C}=3$.

a large amount of scattering data [7,9] and then the spectral functions are predicted. It is also worth pointing out that we have successfully generated ten relevant resonances below $1.5 \mathrm{GeV}: f_{0}(500)$, $f_{0}(980), f_{0}(1370), a_{0}(980), a_{0}(1450), K_{0}^{*}(800), K_{0}^{*}(1430), \rho(770), K^{*}(892)$ and $\phi(1020)$, which are compatible with their PDG values [2]. The resulting scalar spectral functions with the physical masses of light pseudoscalar mesons are shown in Fig. 1. Several interesting features are observed from this plot. First we find clear bumps contributed by the $\sigma$ or $f_{0}(500)$ resonance in the region below 0.5 $\mathrm{GeV}^{2}$ for the $a=0,8$ cases. This is a remarkable result by taking into account that the $f_{0}(500)$ is a very broad resonance, namely with a pole around $440-250 i$ in the complex energy plane. This effect is already known in $D \rightarrow \pi \pi \pi$ decays [14]. On the contrary, we find instead that the narrower states (compared to the $\sigma) f_{0}(1370)$ and $a_{0}(1450)$ manifest themselves as plateaus in the $a=8$ and $a=3$ cases, respectively. This information is quite precious, since we give a concrete example where resonances and background (here it corresponds to meson-meson non-resonant effects) can not be simply described as a peak plus some noise because interferences are quite strong. Interestingly we find that meson-meson non-resonant effects are clearly reduced in the chiral limit, as shown in the left panel of Fig. 2, and they completely disappear in the large $N_{C}$ and chiral limits, right panel of Fig. 2.

In order to study the sum rules in Eq. (5), we need to integrate the spectral functions up to $s_{0}$ and for later convenience we define $W_{i}=16 \pi \int_{0}^{s_{0}} \operatorname{Im} \Pi_{i}(s) d s$ with $i=S^{8}, S^{0}, S^{3}, P^{0}, P^{8}, P^{3}$. The results for three different values of $s_{0}$ are given in Table 1 together with the average values and variances of the different $W_{i}$. The numbers in the last column of Table 1 represent the degrees of the violation of the sum rules. Therefore we conclude that the scalar and pseudoscalar spectral function sum rules are satisfied in the chiral limit, with a violation at most around $10 \%$.

\begin{tabular}{|c|c|c|c|c|c|c|c|c|c|}
\hline & $W_{S^{0}}$ & $W_{S^{8}}$ & $W_{S^{3}}$ & $W_{P^{0}}$ & $W_{P 8}$ & $W_{P^{3}}$ & $\bar{W}$ & $\sigma_{W}$ & $\sigma_{W} / \bar{W}$ \\
\hline Physical masses & $\begin{array}{lll}8.6 & 9.0 & 9.6\end{array}$ & $\begin{array}{lll}7.4 & 7.5 & 7.7\end{array}$ & $\begin{array}{lll}7.0 & 7.2 & 7.4\end{array}$ & 8.9 & 11.3 & 10.1 & 9.0 & 1.5 & 0.16 \\
\hline$m_{q}=0$ & $\begin{array}{lll}6.9 & 7.0 & 7.1\end{array}$ & $\begin{array}{|lll|}6.8 & 7.0 & 7.3\end{array}$ & $\begin{array}{lll}6.6 & 6.8 & 7.0\end{array}$ & 5.5 & 7.4 & 7.4 & 6.9 & 0.7 & 0.10 \\
\hline
\end{tabular}

Table 1. Results from the integration of the spectral functions from 0 up to $s_{0}$, with $s_{0}=2.5,3,3.5 \mathrm{GeV}^{2}$.

\section{Acknowledgements}

Z.H.G would like to thank the organizers of MESON2012 to provide the nice atmosphere and enjoyable social events during the conference. This work is partially funded by the grants MEC FPA2010- 

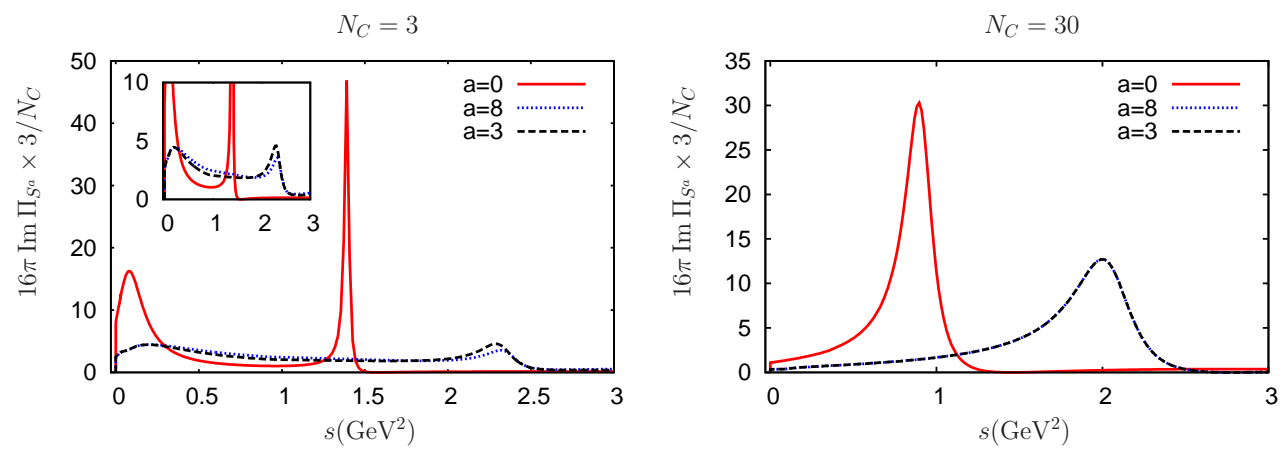

Fig. 2. Scalar spectral functions in the chiral limit for $N_{C}=3$ (left) and $N_{C}=30$ (right).

17806 and the Fundación Séneca 11871/PI/09. We also thank the financial support from the BMBF grant 06BN411, the EU-Research Infrastructure Integrating Activity "Study of Strongly Interacting Matter" (HadronPhysics2, grant No. 227431) under the Seventh Framework Program of EU and the Consolider-Ingenio 2010 Programme CPAN (CSD2007-00042). Z.H.G. acknowledges CPAN postdoc contract in the Universidad de Murcia and financial support from the grants National Natural Science Foundation of China (NSFC) under contract No. 11105038, Natural Science Foundation of Hebei Province with contract No. A2011205093 and Doctor Foundation of Hebei Normal University with contract No. L2010B04.

\section{References}

1. M. A. Shifman, A. I. Vainshtein and V. I. Zakharov, Nucl. Phys. B 147 (1979) 385; Nucl. Phys. B 147 (1979) 448.

2. J. Beringer et al. [Particle Data Group Collaboration], Phys. Rev. D 86 (2012) 010001.

3. S. Weinberg, Physica A 96 (1979) 327; J. Gasser and H. Leutwyler, Annals Phys. 158 (1984) 142; Nucl. Phys. B 250 (1985) 465.

4. J. A. Oller and E. Oset, Phys. Rev. D 60 (1999) 074023.

5. U. -G. Meissner and J. A. Oller, Nucl. Phys. A 679 (2001) 671.

6. J. A. Oller and E. Oset, Nucl. Phys. A 620 (1997) 438 [Erratum-ibid. A 652 (1999) 407]; L. Roca, E. Oset and J. Singh, Phys. Rev. D 72 (2005) 014002; Z. -Y. Zhou and Z. Xiao, Phys. Rev. D 83 (2011) 014010; F. -K. Guo, et al., Phys. Lett. B 641 (2006) 278; R. Garcia-Martin, et al., Phys. Rev. Lett. 107 (2011) 072001; L. Y. Dai, X. G. Wang and H. Q. Zheng, Commun. Theor. Phys. 57 (2012) 841; M. Albaladejo and J. A. Oller, Phys. Rev. Lett. 101 (2008) 252002.

7. Z. -H. Guo and J. A. Oller, Phys. Rev. D 84 (2011) 034005.

8. Z. -H. Guo, J. A. Oller and J. Ruiz de Elvira, Phys. Lett. B 712 (2012) 407.

9. Z. -H. Guo, J. A. Oller and J. Ruiz de Elvira, arXiv:1206.4163 [hep-ph], to appear in Phys. Rev. D.

10. Z. -H. Guo, J. Prades and J. A. Oller, Nucl. Phys. Proc. Suppl. 207-208 (2010) 184; Z. -H. Guo and J. A. Oller, eConf C110613 (2011) [arXiv:1108.5865 [hep-ph]]; Z. -H. Guo, J. A. Oller and J. R. de Elvira, PoS QNP 2012 (2012) 066.

11. P. Di Vecchia and G. Veneziano, Nucl. Phys. B 171 (1980) 253; C. Rosenzweig, J. Schechter and T. Trahem, Phys. Rev. D 21 (1980) 3388; E. Witten, Ann. Phys. 128 (1980) 363; K. Karawabayashi and N. Ohta, Nucl. Phys. B 175 (1980) 477; Prog. Theor. Phys. 66 (1981) 1789.

12. G. Ecker, J. Gasser, A. Pich and E. de Rafael, Nucl. Phys. B 321 (1989) 311.

13. M. Jamin and M. Munz, Z. Phys. C 60 (1993) 569.

14. J. A. Oller, Phys. Rev. D 71 (2005) 054030. 